\title{
BMJ Open Mixed-method study to assess the feasibility, acceptability and early effectiveness of the Hospital to Home programme for follow-up of high-risk newborns in a rural district of Central Uganda: a study protocol
}

Daniel Kabugo, ${ }^{1}$ Heidi Nakamura, ${ }^{2}$ Brooke Magnusson, ${ }^{2}$ Madeline Vaughan, ${ }^{3}$ Beatrice Niyonshaba, ${ }^{1}$ Cornety Nakiganda, ${ }^{1}$ Christine Otai, ${ }^{1}$ Kimber Haddix-McKay, ${ }^{2,4}$ Margaret Seela, ${ }^{5}$ Joyce Nankabala, ${ }^{5}$ Josephine Nakakande, ${ }^{5}$ Moses Ssekidde, ${ }^{5}$ Cally J Tann, ${ }^{6,7,8}$ Benjamin J S al-Haddad, ${ }^{9}$ James Nyonyintono, ${ }^{5}$ Paul Mubiri, ${ }^{10}$ Peter Waiswa,,${ }^{11,12,13}$ Mohan Paudel (iD ${ }^{3}$

To cite: Kabugo D, Nakamura $\mathrm{H}$, Magnusson B, et al. Mixedmethod study to assess the feasibility, acceptability and early effectiveness of the Hospital to Home programme for follow-up of high-risk newborns in a rural district of Central Uganda: a study protocol. BMJ Open 2021;11:e043773. doi:10.1136/ bmjopen-2020-043773

- Prepublication history and additional materials for this paper is available online. To view these files, please visit the journal online (http://dx.doi. org/10.1136/bmjopen-2020043773).

DK, HN, BM, MV and MP contributed equally.

Received 02 September 2020 Revised 07 January 2021 Accepted 10 February 2021

A) Check for updates

(C) Author(s) (or their employer(s)) 2021. Re-use permitted under CC BY-NC. No commercial re-use. See rights and permissions. Published by BMJ.

For numbered affiliations see end of article.

Correspondence to

Dr Mohan Paudel;

mohan.paudel@adaragroup.org

\section{ABSTRACT}

Introduction A follow-up programme designed for high-risk newborns discharged from inpatient newborn units in lowresource settings is imperative to ensure these newborns receive the healthiest possible start to life. We aim to assess the feasibility, acceptability and early outcomes of a discharge and follow-up programme, called Hospital to Home $(\mathrm{H} 2 \mathrm{H})$, in a neonatal unit in central Uganda.

Methods and analysis We will use a mixed-methods study design comparing a historical cohort and an intervention cohort of newborns and their caregivers admitted to a neonatal unit in Uganda. The study design includes two main components. The first component includes qualitative interviews ( $\mathrm{n}=60$ or until reaching saturation) with caregivers, community health workers called Village Health Team (VHT) members and neonatal unit staff. The second component assesses and compares outcomes between a prospective intervention cohort ( $n=100$, born between July 2019 and September 2019) and a historical cohort $(n=100$, born between July 2018 and September 2018) of infants. The historical cohort will receive standard care while the intervention cohort will receive standard care plus the $\mathrm{H} 2 \mathrm{H}$ intervention. The $\mathrm{H} 2 \mathrm{H}$ intervention comprises training for healthcare workers on lactation, breast feeding and neurodevelopmentally supportive care, including cue-based feeding, and training to caregivers on recognition of danger signs and care of their high-risk infants. Infants and their families receive home visits until 6 months of age, or longer if necessary, by specially trained VHTs. Quantitative data will be analysed using descriptive statistics and regression analysis. All results will be stratified by cohort group. Qualitative data will be analysed guided by Braun and Clarke's thematic analysis technique.

Ethics and dissemination This study protocol was approved by the relevant Ugandan ethics committees. All participants will provide written informed consent. We will

\section{Strengths and limitations of this study}

- This study uses mixed-method approach to examine feasibility and acceptability of a novel, familycentred discharge and follow-up programme for high-risk newborns in a low-resource setting.

- The quantitative tools and qualitative guides for this study have been thoroughly prepared in consultation with programme experts, research scholars and academics.

- The qualitative aspects of feasibility and acceptability will be examined using the rich experiences of a range of participants: mothers from local communities, community health workers and nurses and medical officers working in a neonatal unit.

- The quantitative data from the study will be used to assess the health and neurodevelopmental outcomes of high-risk newborns after discharge from a neonatal unit, which, as of now, remain largely unknown.

- While the study design and small sample size may not provide definitive powered estimates on impacts of the intervention, it will indicate whether a further large-scale study is warranted in the future.

disseminate through peer-reviewed publications and key stakeholders and public engagement.

Trial registration number ISRCTN51636372; Pre-result.

\section{INTRODUCTION}

Background

Globally, it is estimated that approximately 7000 newborn babies die every day, which constitutes nearly half of all under-five deaths 
and $62 \%$ of infant deaths. ${ }^{1}$ Additionally, an estimated 19 million newborns are born prematurely or with other life-threatening conditions every year. ${ }^{2}$ Over $90 \%$ of these high-risk newborns are from developing countries in South Asia and sub-Saharan Africa. ${ }^{2}$ Newborns who are preterm, low birth weight or who develop illnesses early in life, often face significant nutrition and developmental challenges. ${ }^{3}$ While mortality rates for high-risk newborns are declining due to improvements in newborn care, it has not reduced the prevalence of disabilities among this group at the same rate. ${ }^{4}$ Surviving infants are at high risk of low-severity neurological impairments including learning disabilities, low or borderline IQs, attention deficit hyperactivity disorder, autism spectrum disorders, poor social-emotional competence and stunted cognitive, language and motor skills. ${ }^{5}$

Additionally, high-risk newborns admitted to a neonatal unit experience a much higher rate of hospital readmission and death during their first year of life compared with healthy, term newborns. ${ }^{6} 7$ Careful preparation of the family for discharge and appropriate follow-up after discharge may reduce these risks. ${ }^{7-9}$ Despite an increasing number of neonatal units being established in low-resource settings,${ }^{10-13}$ very few follow-up programmes exist to ensure that high-risk infants receive the ongoing support and care they require after discharge. ${ }^{347-914}$ As a result, there is a dearth of evidence on the outcomes of these infants once they go home. Adara Development analysed a year of data before the intervention (year 2017) and found that merely one-third of newborns discharged from the neonatal unit came back to Kiwoko Hospital for their first 2-week follow-up appointment. By month 4, fewer than 1 in 10 attended follow-up appointments, leaving the outcomes of these babies unknown. It is widely recognised that there are serious barriers to families accessing facility-based follow-up care for infants, including a global shortage of neonatal nurses, weak referral systems, lack of transportation, poverty, cost of care, lack of facilities, weak empowerment of women and lack of education and awareness of danger signs in parents and clinicians. ${ }^{1415}$ These evidences indicate that follow-up of high-risk newborns in low-resource settings requires a coordinated system for linking the facility with the health workers in the community. ${ }^{6}$ Follow-up care is critical because it can detect early danger signs and feeding difficulties that could lead to stunting, wasting or failure to thrive or death, and can be a vehicle for early identification of neurodevelopmental delays. ${ }^{4-9}$ Early detection and intervention with locally appropriate, wellfunctioning follow-up programmes is essential for giving these high-risk infants the best opportunity to reach their full potential. ${ }^{6}$

\section{Aim}

The study aims to evaluate whether a family-centred, facility and community-based discharge and follow-up programme for newborns admitted to a low-resource neonatal unit is feasible, acceptable and can provide early evidence of impact on growth, neurodevelopment and infant-caregiver attachment, compared with current standard care practices.

\section{Objectives and hypotheses}

The objectives of the study are to:

1. Evaluate the feasibility and acceptability of the Hospital to Home $(\mathrm{H} 2 \mathrm{H})$ programme.

Hypothesis: The H2H programme is feasible to implement and is acceptable to both the clients (caregivers) and service providers (Village Health Teams (VHTs) and hospital staff).

2. Obtain preliminary data on whether the $\mathrm{H} 2 \mathrm{H}$ programme improves infant health and well-being and infant-caregiver attachment at 6 months corrected age (corrected age refers to the infant's chronological age minus the number of weeks born prematurely) when compared with a historical cohort who did not receive the programme.

Hypothesis: Infants receiving the $\mathrm{H} 2 \mathrm{H}$ programme demonstrate reduced rates of undernutrition (stunting, wasting, underweight); increased head circumference; increased neurodevelopmental scores on the Griffith Mental Development Scales (GMDS); and increased scores on the Maternal Infant Responsiveness Instrument (MIRI).

\section{METHODS}

We will use a mixed-method study design. Objective 1 will be assessed using an observational descriptive design and Objective 2 will be assessed with a post-test only design ${ }^{16}$ (figure 1).

\section{Study setting}

\section{Overview of Uganda}

Uganda is ranked 159 of 189 in the 2019 Human Development Index. ${ }^{17}$ Subsistence farming is the major source of income for the majority of people and more than a quarter live below the poverty line. ${ }^{18}$ Uganda is still grappling with high maternal (336 per 100000 live births) and neonatal (27 per 1000 live births) mortality rates. ${ }^{19}$ Neonatal deaths account for $42 \%$ of the under-five deaths and over $60 \%$ of the total infant deaths in the country. ${ }^{20}$ It is estimated that Uganda has nearly $7 \%$ preterm birth rate, which amounts to 108000 babies born too soon each year; 9800 direct preterm deaths and 5700 impaired preterm survivors per year. ${ }^{21}$

\section{Kiwoko Hospital}

The $\mathrm{H} 2 \mathrm{H}$ intervention will take place at Kiwoko Hospital (KH) based in the Nakaseke district and in two surrounding districts-Luwero and Nakasongola (figure 2). Socio-economically, there are no marked differences across these three districts; they represent rural Uganda and a region with relatively poor socioeconomic circumstances. These districts are inhabited by people of two major tribes-Baganda and Banyakore who are mostly farmers and pastoralists by their occupation. 


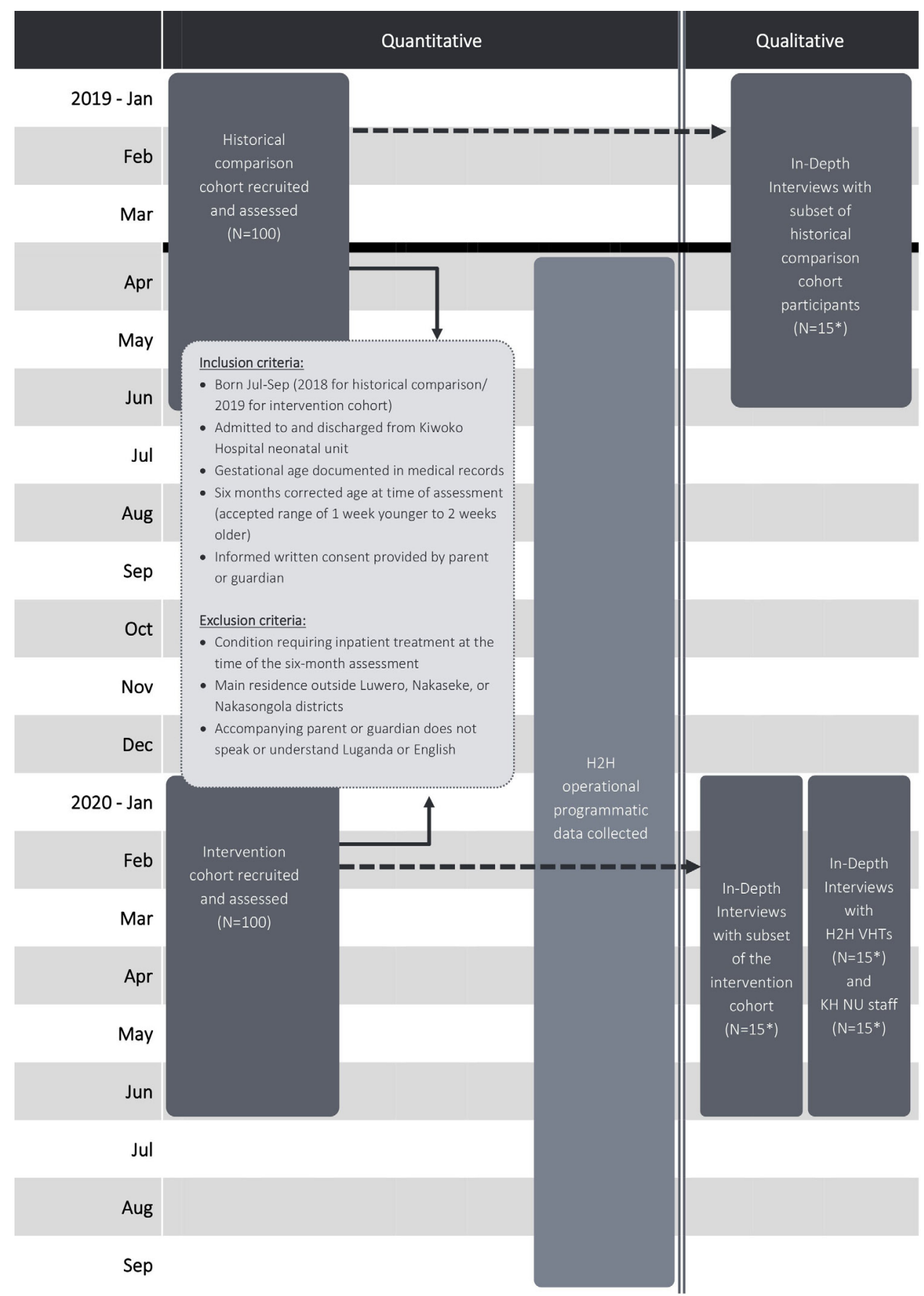

Figure $1 \mathrm{H} 2 \mathrm{H}$ study flow chart. *Or until saturation has been reached. $\mathrm{H} 2 \mathrm{H}$, Hospital to Home; $\mathrm{KH}$, Kiwoko Hospital; $\mathrm{NU}$, neonatal unit, VHTs, Village Health Teams.

KH is a rural faith-based hospital located in the Nakaseke district of Central Uganda. The 250-bed hospital sees over 40000 patients a year and serves a catchment area of approximately 1 million people. For the past 22 years, the study implementer, Adara Development, has worked alongside $\mathrm{KH}$ to develop maternal, newborn and child health services, including the establishment of a neonatal unit that now provides care to over 1200 newborns a year. ${ }^{22}$ Of the 1311 newborns admitted to the unit in 2019, 52\% are low birth weight $(<2.5 \mathrm{~kg}), 14 \%$ very low birth weight $(<1.5 \mathrm{~kg})$ and $3 \%$ extremely low birth weight $(<1 \mathrm{~kg}) .^{22}$
The KH neonatal unit provides thermal care, kangaroo care, assisted feeding, intravenous therapy, oxygen therapy, phototherapy, bubble continuous positive airway pressure, seizure management and management of infections, among other services. In the community, KH runs community-based healthcare programmes which include safe motherhood outreach clinics providing antenatal and postnatal care, mother and child immunisations, health education and family planning services. The clinics are supported by a network of VHTs who provide health education and mobilise the community to attend clinics. 


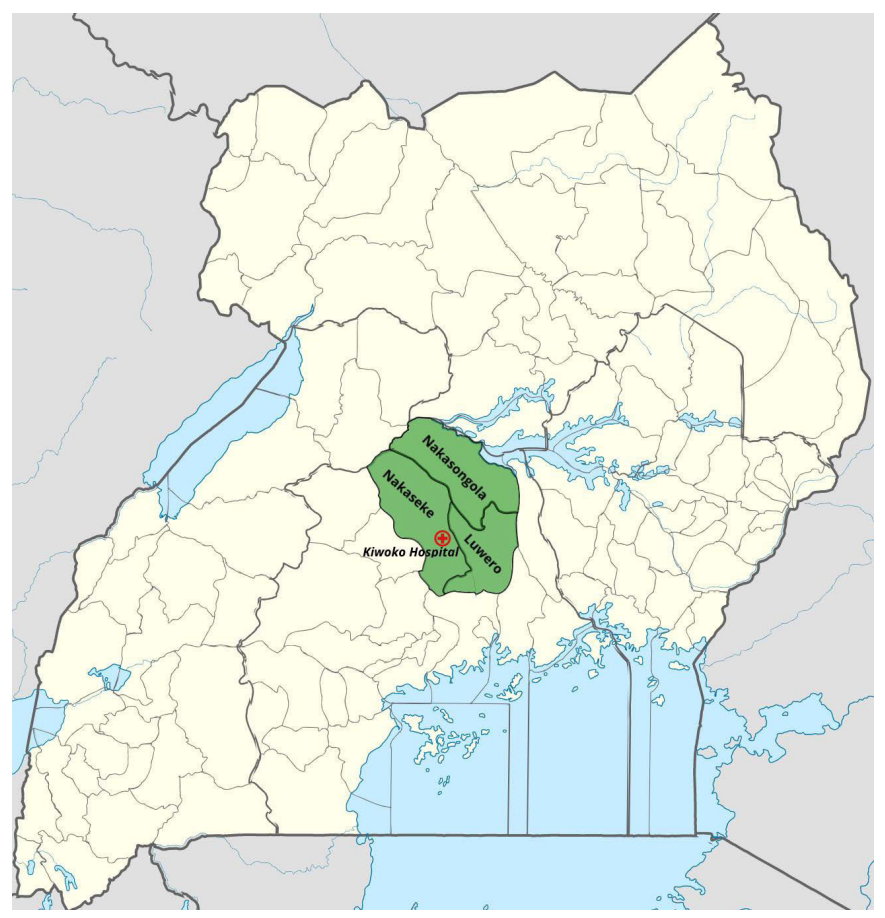

Figure 2 Map showing Hospital to Home study districts.

\section{Study population, sample size and recruitment}

The study population will be selected from high-risk newborns and their caregivers discharged from the neonatal unit at $\mathrm{KH}$ and who live in Luwero, Nakaseke or Nakasongola districts.

\section{Inclusion and exclusion criteria}

We will use the following inclusion and exclusion criteria to recruit infants for the historical and intervention cohorts (box 1) as well as for the in-depth interview participants (box 2):

\section{Sample size}

To address hypothesis 1 , in-depth interviews (IDI) will be conducted with caregivers or parents $(n=15$ or until reaching saturation) from each cohorts, H2H VHTs $(\mathrm{n}=15$ or until reaching saturation) and staff working in

\section{Box 1 Inclusion and exclusion criteria for infants}

Inclusion criteria
Born between July and September ( 2018 for historical comparison
cohort and 2019 for intervention cohort).
Admitted and discharged from the KH neonatal unit.
Gestational age documented.
Six months corrected age at the time of assessment (accepted
range of 1 week younger to 2 weeks older).
Informed written consent provided by the caregiver.
Exclusion criteria
Condition requiring inpatient treatment at the time of the 6-month
assessment.
Main residence outside Nakaseke, Luwero or Nakasongola districts.
Accompanying caregiver does not speak or understand Luganda or
English.

Box 2 Inclusion and exclusion criteria, in-depth interview participants

Inclusion criteria
A caregiver of a newborn from the historical cohort or prospective
cohort who agreed to be interviewed by the research team.
A nurse or a clinician working in $\mathrm{KH}$ neonatal unit or a VHT mem-
ber or other staff engaged in delivering the Hospital to Home $\mathrm{H} 2 \mathrm{H})$
intervention in communities who agreed to be interviewed by the
research team.
Exclusion criteria
A caregiver who did not belong to either of the two study cohorts and
a VHT member who was not engaged in delivery of $\mathrm{H} 2 \mathrm{H}$ intervention.

the KH neonatal unit ( $\mathrm{n}=15$ or until reaching saturation). Sample sizes have been determined based on an estimate of the number of subjects required to achieve saturation. Sample sizes will vary, depending on when saturation is reached. ${ }^{23}$ No further interviews will be conducted when the research team finds additional interviews adding no new ideas on the aspects of feasibility and acceptability of the $\mathrm{H} 2 \mathrm{H}$ programme.

To address hypothesis 2 , the study will recruit 100 babies in each cohort. The sample size is based on a pragmatic approach and is not powered to detect programme outcomes but will provide important preliminary data and inform an appropriate effect size and sample size for future studies.

\section{Recruitment}

A historical cohort of infants will be selected prior to establishment of $\mathrm{H} 2 \mathrm{H}$ at $\mathrm{KH}$. We will use a random number generator to randomly select 100 infants who were born between July 2018 and September 2018, who received standard care in the $\mathrm{KH}$ neonatal unit and met eligibility criteria as outlined in the study flow chart (see figure 1). For the $\mathrm{H} 2 \mathrm{H}$ intervention cohort, we will use a random number generator to select 100 infants who were born between July 2019 and September 2019 and who received standard care plus $\mathrm{H} 2 \mathrm{H}$ intervention in the $\mathrm{KH}$ neonatal unit. Data from infants in both cohorts will be collected when they reach 6 months corrected age.

The study team will identify all eligible newborns from a database of patients admitted to the KH neonatal unit. Those meeting initial eligibility criteria will be entered into a screening log. When an infant on the screening $\log$ reaches $5 \frac{1}{2}$ to 6 months corrected age, study staff will contact caregivers of the infant over the phone to introduce them to the research study. They will be invited to come to $\mathrm{KH}$ with their infant when the infant is 6 months corrected age to attend the $\mathrm{H} 2 \mathrm{H}$ study clinic. Caregivers interested to participate in the research will be provided travel incentive to reach up to the $\mathrm{KH} \mathrm{H} 2 \mathrm{H}$ clinic.

When infants and their caregivers come to the $\mathrm{H} 2 \mathrm{H}$ study clinic, the caregiver will go through the informed consent process with a trained staff member. Once a 
caregiver has provided consent for their participation in the study, study assessments will take place.

\section{About the $\mathrm{H} 2 \mathrm{H}$ intervention}

$\mathrm{H} 2 \mathrm{H}$ is a family-centred programme of facility-based care and at-home follow-up of high-risk newborns discharged from the KH Neonatal Unit. Caregivers and their infants admitted to the $\mathrm{KH}$ Neonatal Unit will receive the $\mathrm{H} 2 \mathrm{H}$ facility-based component during their hospital stay and before being discharged home. Discharge planning begins early on admission into the neonatal unit by educating and preparing the family to be able to care for their infant safely at home. When the discharge date nears, the neonatal unit team coordinates with a VHT working within the family's geographical area. This VHT will be assigned to the family to provide in-home follow-up care and support after discharge. To improve programme adherence in the community component of the intervention, VHTs will be regularly supervised by a research community midwife, provided monetary (travel and airtime) incentive and non-monetary incentives including a bicycle and supplies for their follow-up visits. To improve programme adherence in the facility component of intervention, ongoing education will be provided to staff and experts will be available to answer questions via a messaging application and email. The programme is described in box 3 .

\section{Outcomes}

Feasibility and acceptability of the $\mathrm{H} 2 \mathrm{H}$ programme will be evaluated with a mixed-method approach using quantitative data collected in the hospital and during at-home visits, and qualitative data from IDIs. Preliminary evidence of impact will be evaluated quantitatively using data from the historical comparison and intervention cohorts. Infants in each cohort will be assessed at the study clinic when they are 6 months corrected age and assessments will be conducted by trained study staff through observation and anthropometric assessments on infants, and structured interview questionnaires with caregivers.

The key outcome measures of the study will be:

1. Feasibility of the H2H facility component as assessed by the percentage of infants discharged from the $\mathrm{KH}$ neonatal unit meeting discharge criteria and percentage of caregivers receiving the discharge teaching topics (online supplemental additional files 3 and 4 ). This operational programme data will be collected by KH staff. Qualitative tools will also be used to capture additional information on feasibility of the facility component.

2. Feasibility of the $\mathrm{H} 2 \mathrm{H}$ community component as assessed by the percentage of completed scheduled visits from VHTs and the percentage of infants who sought medical care after being referred by a H2H VHT. This operational programme data will be collected by $\mathrm{H} 2 \mathrm{H}$ VHTs (online supplemental additional file 1). Qualitative tools will also be used to capture additional information on feasibility of the community component.
Box 3 Hospital to Home programme components

\section{Facility components}

- Training for KH neonatal unit staff in neurodevelopmentally supportive care practices for sick and premature infants (including cuebased feeding), as well as breast feeding and lactation support for mothers.

- Addition of a neonatal therapist to provide neurodevelopmentally supportive care to the patients in the neonatal unit.

- Implementation of improved discharge processes and coordination of care in the KH neonatal unit. This will include an individualised care plan for each patient, with the aim of reducing the length of hospital stay, decreasing readmissions and improving neonatal outcomes. This will be facilitated by the addition of two part-time 'discharge coordinators' to oversee and manage this process.

- Implementation of a family-centred programme that encourages parents to learn about and participate in their infant's care. An education programme and daily classes will teach parents about the care of their infant. These discharge teaching topics include lactation and breast feeding; kangaroo mother care/temperature regulation, bathing, skin and cord care; immunisations; recognition of newborn danger signs and the importance of care seeking; family planning; mother's nutrition; safe sleep; safety at home; sanitation and prevention of infection at home; and child development.

- Implementation of a system to clearly link eligible infants with a network of $\mathrm{H} 2 \mathrm{H}$ VHTs, to provide follow-up care and parental support after discharge from the neonatal unit.

- Introduction of a set of discharge criteria to ensure infants are ready to return home. This includes ensuring the infant is physiologically mature and maintains stable cardiorespiratory status; takes full feeding amount without cardiorespiratory compromise; weighs $>1.5 \mathrm{~kg}$ with consistent weight gain; maintains normal temperature in a cot; and shows no clinical signs of convulsions.

\section{At-home follow-up components}

- Training of $100 \mathrm{H} 2 \mathrm{H}$ VHTs. VHTs will receive specialised training in the care of high-risk infants, including topics such as recognising newborn danger signs, lactation and breast feeding support, monitoring weight and growth, child development and the importance of care seeking (online supplemental additional files 1 and 2).

- Formation of VHT teams, consisting of 10-12 VHTs who are led by one 'Chief VHT'.

- Addition of a $\mathrm{H} 2 \mathrm{H}$ community midwife who will support the teams of VHTs by providing supportive supervision. VHT teams will meet monthly for additional support and training.

- Implementation of at-home follow-up visits with families after discharge from the KH neonatal unit from assigned VHTs.

- Training for VHTs on the process for referring babies who require additional care, including referrals to speciality services, when necessary.

3. Acceptability of the $\mathrm{H} 2 \mathrm{H}$ programme as assessed by the percentage of families that refuse follow-up visits from VHTs. Qualitative tools will also be used to capture information on acceptability.

4. Preliminary evidence of impact on growth as assessed by the rates of undernutrition (stunting, wasting, underweight) and nutritional status as indicated by head circumference and middle upper arm circumference. These data will be collected by anthropometric assessment of infants in both cohorts (online supplemental 
additional file 5). Additional operational programme data for the intervention cohort will be collected by H2H VHTs (online supplemental additional file 1).

Other outcomes of interest:

5. Rates of exclusive breast feeding as assessed by percentage of infants exclusively breast fed for 6 months (online supplemental additional file 6).

6. Rates of immunisation as assessed by the rates of infants receiving the recommended immunisations by 6 months (online supplemental additional file 6).

7. Infant development as assessed by using the standard Griffith Mental Development Scales ${ }^{24}$ (online supplemental additional file 5).

8. Child-caregiver attachment as assessed using the already established Maternal Infant Responsiveness Instrument ${ }^{25}$ translated into local Luganda language (online supplemental additional file 7).

9. VHT knowledge and skills as assessed by knowledge tests administered to VHTs after initial training and at ongoing intervals to assess knowledge gained and retained. This will also be assessed through a skills assessment of a random sample of VHTs to determine their adherence to home visit protocols. Research staff (a community midwife) will conduct the assessments (online supplemental additional files 8 and 9).

10. VHT engagement as assessed by the attendance rate at monthly VHT meetings. This data will be completed by the $\mathrm{H} 2 \mathrm{H}$ community midwife. Qualitative tools will also be used to capture information on VHT engagement.

11. Length of hospital stay as assessed by the duration of time infants stay in the $\mathrm{KH}$ neonatal unit prior to discharge.

\section{Qualitative data}

IDIs will be conducted with three different populations: KH neonatal unit staff, VHTs and caregivers of newborns from the historical and intervention cohorts (online supplemental additional file 10). IDIs with $\mathrm{KH}$ staff and VHTs will be conducted 12-18 months after H2H programme implementation. They will include questions related to feasibility and acceptability of delivering the $\mathrm{H} 2 \mathrm{H}$ programme in the hospital and at home; appropriateness of the programme; and perceived challenges of the programme. IDIs with caregivers of infants in each cohort will be conducted after the infants' assessment at 6 months corrected age. These interviews will include questions related to their experience during their hospital stay and caring for their infant after discharge. Caregivers in the intervention cohort will be asked additional questions related to their acceptance of the $\mathrm{H} 2 \mathrm{H}$ programme.

Interviews will be audio-recorded and transcribed. Those conducted in the local language, Luganda, will be transcribed and translated into English by a trained transcriptionist and translator. Transcripts will be uploaded and managed in NVivo V.12.00. The interviews will be conducted by trained study staff who have experience in qualitative research.

\section{Data management}

Operational programmatic data from the $\mathrm{H} 2 \mathrm{H}$ facility component will be collected by $\mathrm{KH}$ neonatal unit staff as part of standard $\mathrm{H} 2 \mathrm{H}$ inpatient process. Operational programmatic data from the $\mathrm{H} 2 \mathrm{H}$ community component will be collected by H2H VHTs as part of at-home visits, and by the community midwife. Data from IDIs and interviews will be collected in the form of audiotape and field notes. Data from the historical comparison and intervention cohorts will be collected with infants that are 6 months corrected age, by trained study staff. Data will be collected by hand on paper data collection forms and entered electronically by a trained data entry team into a REDCap cloud-based electronic database. Paper forms will be stored in a locked cabinet at the site. The database will be maintained on a secure server and regularly backed up. Access to the locked cabinet and electronic database will be limited to essential individuals. Data entry will be overseen by the monitoring and evaluation officer. Study data will be de-identified whenever possible. No names of participants will be published or made publicly available.

\section{Participant and public involvement}

The H2H intervention, design and conduct were shaped after consultation with service providers from the neonatal unit and the Community Based Health Care Department of Kiwoko Hospital. The study team orally presented the $\mathrm{H} 2 \mathrm{H}$ pilot programme concept to the Uganda Ministry of Health and the Uganda National Newborn Steering Committee. Our plan to disseminate findings will engage local and national key stakeholders, including parents, VHTs, KH staff and local district health offices.

\section{Analysis}

The first outcome, feasibility of the H2H facility component, will be assessed by summarising the percentage of infants during the intervention period who met the discharge criteria and percentage of infants whose caregiver received the discharge teaching topics. Feasibility will be demonstrated if $70 \%$ of infants meet discharge criteria and $70 \%$ of parents receive the discharge teaching topics.

The second outcome, feasibility of the H2H community component, will be assessed by summarising the number of at-home follow-up visits completed per infant. Mean, median, range and IQR will be calculated. Feasibility will be demonstrated if the infants receive $60 \%$ of the recommended at-home follow-up visits. The schedule for home follow-up visits is determined by the infants' level of risk and weight at discharge and is further guided by an ongoing assessment of risk and weight gain until they reach 6 months corrected age. Feasibility of the $\mathrm{H} 2 \mathrm{H}$ community component will also be demonstrated if $60 \%$ of infants sought medical care at a health facility if referred by a VHT.

The third outcome, acceptability of the H2H programme, will be assessed by summarising the percentage of caregivers that refuse the community component of the 
intervention. Acceptability will be demonstrated if $15 \%$ or fewer caregivers refuse the community component of the intervention.

The fourth outcome, preliminary evidence of impact on growth, and secondary outcomes $5-8$, will be assessed by comparing outcomes between the historical and intervention cohorts. Infants in each cohort will be assessed when they are 6 months corrected age. We will summarise outcome measures by cohort group. These outcome data will be analysed by descriptive statistics and linear regression. Quantitative data collected in a REDCap database will be analysed in Excel, SPSS V.26.00, Stata 15.00 and R.

Outcomes 9 and 10 will be analysed by summarising mean knowledge and skill scores and percentage of VHTs who score $80 \%$ or more on both knowledge and skills domains. Composite scores on knowledge and skills will be computed by summing up all knowledge and skill items as outlined in the assessment form.

Outcome 11 will be analysed by summarising the average length of stay for infants in both historical and intervention cohort.

Qualitative aspects of feasibility and acceptability, including operational challenges in programme implementation and caregivers' and staff's comment on intervention satisfaction, will complement quantitative markers of feasibility and acceptability. Qualitative data will be analysed following a thematic analysis approach, using a comprehensive coding process as guided by Braun and Clarke's thematic analysis technique. ${ }^{26}$ Themes will be based on the study objectives and those emerging from the data. Social scientists (three people) will agree the coding frame and undertake analysis collaboratively to ensure agreement on the coding approach. Thematic summaries will be developed and shared with the wider team for discussion.

\section{ETHICS AND DISSEMINATION \\ Ethics}

This study protocol has been approved by the Makerere University School of Public Health Institutional Review Board (Protocol number 629) and Uganda National Council for Science and Technology (UNCST) (Reference number HS292ES). We have also obtained local approval from District Health Offices in the three surrounding districts: Nakaseke, Nakasongola and Luwero. Written informed consent will be obtained from all study participants (online supplemental additional file 11). The infant's parent or guardian will provide consent to participate in the research, as well as for having their infant assessed, for the use of existing hospital and programme data, and for participation in qualitative interviews.

Infants recruited into the historical comparison cohort will have received standard care, while infants in the intervention cohort will have received standard care plus the H2H intervention. We believe this study to be lowrisk and believe that the benefits for this population far outweigh the minor risks to the study participants. In the case of the death of an infant, the parents or guardians of the deceased infant might incur some mental stress while being interviewed. We plan to reduce this mental stress by using study staff who have had bereavement training and experience. There is minor risk of mental stress for participants during questions about their personal and health status. Respondents will be frequently reminded of their choice to refuse to answer any questions at any time.

\section{Dissemination}

The study is registered in ISRCTN registry. The study team orally presented the $\mathrm{H} 2 \mathrm{H}$ pilot programme to the Uganda Ministry of Health and the Uganda National Newborn Steering Committee. On completion of the study, we plan to disseminate findings with local and national key stakeholders, including parents, KH staff, local district health offices, Ugandan Ministry of Health and Uganda National Newborn Steering Committee. Our study team plans to publish findings on programme feasibility and acceptability, and early evidence on infant growth and developmental outcomes.

\section{Author affiliations}

${ }^{1}$ Adara Development (Uganda), Adara Group, Nakaseke, Uganda

${ }^{2}$ Adara Development (USA), Adara Group, Edmonds, Washington, USA

${ }^{3}$ Adara Development (Australia), Adara Group, Sydney, New South Wales, Australia

${ }^{4}$ School of Public and Community Health Sciences, University of Montana, Missoula,

Montana, USA

${ }^{5}$ Kiwoko Hospital, Kiwoko, Uganda

${ }^{6}$ Department of Infectious Disease Epidemiology, London School of Hygiene and Tropical Medicine, London, UK

${ }^{7}$ Social Aspects of Health Programme, MRC/UVRI \& LSHTM Uganda Research Unit, Entebbe, Uganda

${ }^{8}$ Neonatal Medicine, University College London Hospitals NHS Trust, London, UK ${ }^{9}$ Division of Neonatology, Department of Pediatrics, University of Washington, Seattle, Washington, USA

${ }^{10}$ School of Public Health, Makerere University, College of Health Sciences, Kampala, Uganda

${ }^{11}$ Department of Health Policy Planning and Management, Makerere University School of Public Health, Kampala, Uganda

${ }^{12}$ Department of Global Public Health, Karolinska Institutet, Stockholm, Sweden ${ }^{13}$ Makerere University Centre of Excellence for Maternal Newborn and Child Health, Makerere University School of Public Health, Kampala, Uganda

Acknowledgements The authors would like to thank the funder, Grand Challenges Canada, Saving Brains for their financial support for the H2H study. The funder approves open access publication per BMJ requirements. The study team provides its sincere gratitude to the members of the Hospital to Home advisory panel, and the Uganda Ministry of Health and National Newborn Steering Committee for their comments, suggestions and support of this pilot study. We are grateful to the team at Kiwoko Hospital and at Adara Development (Uganda, Australia and USA) for carrying the implementation of $\mathrm{H} 2 \mathrm{H}$ forward. We also acknowledge the District Health Offices of Nakaseke, Luwero, Nakasongola and ethics committees of Makerere University School of Public Health and Uganda National Council for Science and Technology for their collaboration and ethical approval of the project.

Contributors DK and HN are the principal investigators of the study. MP drafted the first manuscript. HN, BM, MV, DK and BN provided substantial contribution to write and revise the manuscript draft. PW and JN provided their critical comments throughout from the inception of the pilot to the final production of the manuscript. HN, BM, MV and MP contributed producing study tools. CN, CO, MS, JNan, JNak, JNy, KH-M, PW, PM, CJT, BJSa-H and MS reviewed and provided their critical comments to revise the manuscript. PM and BJSa-H provided statistical expertise. All authors reviewed and agreed the final version of the manuscript.

Funding This work was supported by Saving Brains, Grand Challenges Canada, grant number SB-POC-1810-20757. Saving Brains is a partnership 
of Grand Challenges Canada, Aga Khan Foundation Canada, the Bernard van Leer Foundation, the Bill \& Melinda Gates Foundation, The ELMA Foundation, Grand Challenges Ethiopia, the Maria Cecilia Souto Vidigal Foundation, the Palix Foundation, the UBS Optimus Foundation and World Vision Canada. The trial sponsor is Adara Development (Uganda). Contact name: Daniel Kabugo; PO Box 116, Luwero, Nakaseke District, Uganda; E-mail: daniel.kabugo@adaragroup.org Tel +256 772643234 . BA is supported by T32 GM008244 from the US National Institute of General Medical Sciences.

Disclaimer Grand Challenges Canada is not involved in any way in the preparation and submission of this study protocol. The names and ages cited in Additional file 2: VHT training manual are fictional.

Map disclaimer The depiction of boundaries on this map does not imply the expression of any opinion whatsoever on the part of BMJ (or any member of its group) concerning the legal status of any country, territory, jurisdiction or area or of its authorities. This map is provided without any warranty of any kind, either express or implied.

Competing interests None declared.

Patient consent for publication Not required

Provenance and peer review Not commissioned; externally peer reviewed.

Supplemental material This content has been supplied by the author(s). It has not been vetted by BMJ Publishing Group Limited (BMJ) and may not have been peer-reviewed. Any opinions or recommendations discussed are solely those of the author(s) and are not endorsed by BMJ. BMJ disclaims all liability and responsibility arising from any reliance placed on the content. Where the content includes any translated material, BMJ does not warrant the accuracy and reliability of the translations (including but not limited to local regulations, clinical guidelines, terminology, drug names and drug dosages), and is not responsible for any error and/or omissions arising from translation and adaptation or otherwise.

Open access This is an open access article distributed in accordance with the Creative Commons Attribution Non Commercial (CC BY-NC 4.0) license, which permits others to distribute, remix, adapt, build upon this work non-commercially, and license their derivative works on different terms, provided the original work is properly cited, appropriate credit is given, any changes made indicated, and the use is non-commercial. See: http://creativecommons.org/licenses/by-nc/4.0/.

\section{ORCID iD}

Mohan Paudel http://orcid.org/0000-0002-4620-5197

\section{REFERENCES}

1 Hug L, Sharrow D, Zhong K. Levels \& trends in child mortality report 2018. New York: United Nations Children's Fund, 2018.

2 Lawn JE, Blencowe H, Oza S, et al. Every newborn: progress, priorities, and potential beyond survival. Lancet 2014;384:189-205.

3 van den Boogaard W, Zuniga I, Manzi M, et al. How do lowbirthweight neonates fare 2 years after discharge from a lowtechnology neonatal care unit in a rural district hospital in Burundi? Trop Med Int Health 2017;22:423-30.

4 Pickier RH, McGrath JM, Reyna BA, et al. A model of neurodevelopmental risk and protection for preterm infants. Adv Neonatal Care 2013;13:S11-20.
5 Cheong JL, Doyle LW, Burnett AC, et al. Association between moderate and late preterm birth and neurodevelopment and social-emotional development at age 2 years. JAMA Pediatr 2017; 171:e164805.

6 WHO. Survive and thrive: transforming care for every small and sick newborn. Geneva: World Health Organisation, 2019.

7 American Academy of Pediatrics Committee on Fetus and Newborn. Hospital discharge of the high-risk neonate. Pediatrics 2008;122:1119-26.

8 Macho P, Zukowsky K. Individualized developmental care in the NICU: a concept analysis. Adv Neonatal Care 2017;17:162-74.

9 Sherman MP, Shoemaker C. Follow-Up of NICU patient. Pediatrics 2004;114:1377-97.

10 Mbonye AK, Sentongo M, Mukasa GK, et al. Newborn survival in Uganda: a decade of change and future implications. Health Policy Plan 2012;27:iii104-17.

11 WHO \& UNICEF. Reaching the every newborn 2020 targets and milestones: Executive summary, 2020.

12 Dörnemann J, van den Boogaard W, Van den Bergh R, et al. Where technology does not go: specialised neonatal care in resource-poor and conflict-affected contexts. Public Health Action 2017;7:168-74.

13 Zaka N, Alexander EC, Manikam L, et al. Quality improvement initiatives for hospitalised small and sick newborns in low- and middle-income countries: a systematic review. Implement Sci 2018;13:20

14 Kozuki N, Guenther T, Vaz L, et al. A systematic review of community-to-facility neonatal referral completion rates in Africa and Asia. BMC Public Health 2015:15:989.

15 Moxon SG, Lawn JE, Dickson KE, et al. Inpatient care of small and sick newborns: a multi-country analysis of health system bottlenecks and potential solutions. BMC Pregnancy Childbirth 2015;15:S7.

16 Harris AD, McGregor JC, Perencevich EN, et al. The use and interpretation of quasi-experimental studies in medical informatics. $J$ Am Med Inform Assoc 2006;13:16-23.

17 United Nations Development Programme (UNDP). The 2019 Human Development Report : Beyond income, beyond averages, beyond today: Inequalities in human development in the 21st century. New York: United Nations Development Programme (UNDP), 2019.

18 Uganda Bureau of Statistics. Uganda national household survey 2016/2017. Kampala, Uganda: UBOS, 2018.

19 Sensalire S, Isabirye P, Karamagi E, et al. Saving mothers, giving life approach for strengthening health systems to reduce maternal and newborn deaths in 7 scale-up districts in northern Uganda. Glob Health Sci Pract 2019;7:S168-87.

20 Uganda Bureau of Statistics, ICF. Uganda demographic and health survey 2016. Kampala, Uganda: UBOS and ICF, 2018.

21 USAID, PCI, GAPPS, American College of Nurse-Midwives. Every premie scale: Uganda profile of preterm and low birth weight prevention and care, 2019.

22 Adara Group. The art of the possible: operations report 2019. Sydney: Australia Adara Group, 2019.

23 Fusch PI, Ness LL. Are we there yet? Data saturation in qualitative research. Qualitative Report 2015;20:1408.

24 Smith JA, Bidder RT, Gardner SM. Griffiths scales of mental development and different users child care, health and development 1980;6:11-16.

25 Amankwaa LC, Pickler RH, Boonmee J. Maternal responsiveness in mothers of preterm infants. Newborn Infant Nurs Rev 2007;7:25-30.

26 Braun V, Clarke V. Using thematic analysis in psychology. Qual Res Psychol 2006;3:77-101. 